\title{
Complete Shaking Force and Shaking Moment Balancing of the Position-Orientation Decoupled PAMINSA Manipulator
}

\author{
Sébastien Briot and Vigen Arakelian
}

\begin{abstract}
This paper deals with the complete shaking force and shaking moment balancing of the position-orientation decoupled PAMINSA manipulator. The dynamic reaction forces on the manipulator's base are eliminated by making the total mass center of the moving links stationary. The reaction moments on the frame are eliminated by optimal control of the end-effector, which rotates with prescribed velocity. The numerical simulations carried out using ADAMS software demonstrate that the balanced manipulators transmit no inertia loads to their bases.
\end{abstract}

\section{INTRODUCTION}

$\mathrm{T}$ HE problem of mass balancing of mechanisms is of continuing interest to researchers and different approaches and solutions have been developed and documented [1-3]. But despite its long history, mechanism balancing theory continues to develop and new approaches and solutions are constantly being reported. A new field for their application is the design of mechanical systems for fast manipulation, which are very efficient for advanced robotic applications. The cancellation of the shaking force transmitted to the robot frame can be achieved by a traditional approach, which consists of the fixation of the total mass center of the manipulator moving links [4-10]. However, the cancellation of the shaking moment is a more complicated task. The literature review of works on shaking moment balancing shows that the methods can be classified into three principal groups: (i) shaking moment balancing using counter-rotations [11-15], (ii) shaking moment balancing by adding four bar linkages [16-20] and (iii) shaking moment balancing by optimal trajectory planning [21-23].

The aim of the first approach is to connect the counterweights with planetary gear trains, which generate counter-rotations. The disadvantage of such a balancing is the need for connection of gears to the oscillating links. The oscillations of the links of the mechanism will create noise unless expensive anti-backlash gears are used.

The second approach is achieved by building a mechanical system out of modules - four-bar linkages or parallelepiped mechanisms. The linkages with prescribed geometric parameters allow the optimum redistribution of

Manuscript received January 31, 2009.

S. Briot is with the Institut de Recherche en Communication et Cybernétique de Nantes (IRCCyN), Nantes, 44321 France.

V. Arakelian is with the National Institute of Applied Sciences (I.N.S.A.), Rennes, 35043 France (corresponding author: +33223238492; fax: +33223238726; e-mail: vigen.arakelyan@insa-rennes.fr). the moving mass to achieve shaking moment balancing. The originality of such a solution is the balancing of the shaking moment without counter-rotations. However, balancing by using this method is only attained via a considerably complicated design.

In the third approach the shaking moment is balanced through optimum motion planning. In study [21], a 3-DOFs manipulator is developed in order to carry out an in-plan positioning task in which a redundant degree of freedom is used for shaking moment balancing. The drawback of this approach consists of complementary actuation. It should be noted that the shaking moment of a force balanced 2-DOFs manipulator can be successfully cancelled by a complementary actuator mounted on the frame and rotated with prescribed velocity. Another approach based on the optimum motion planning has been developed for the 3-RRR parallel manipulator [22], in which the trajectory in the horizontal plane of the manipulator is imposed. The rotation of the platform is calculated in such a manner that the angular momentum of the parallel mechanism becomes constant. It should be noted that the legs of a force balanced manipulator have important masses and consequently inertia moment. The rotation of the platform is used for compensation of their moments. However, the platform of a 3-RRR parallel manipulator cannot perform a full-cycle rotation, and as a result the rotary acceleration is limited. In our previous study [23] the complete shaking force and shaking moment of the SCARA robot with 4-DOF has been discussed. Reaction moments on the frame of the SCARA robot are eliminated by optimal control of the end-effector, which rotates with prescribed velocity. In the present paper such an approach is used for the balancing of the positionorientation decoupled PAMINSA manipulator.

The paper is organized as follows. At first the concept of the manipulator is discussed. Then the shaking force and shaking moment balancing are considered. In the last part a numerical simulation carried out by using ADAMS software illustrates that the balanced manipulators transmit no inertia loads to their bases.

\section{Position-ORIENTATION DECOUPLED PAMINSA MANIPULATOR WITH 6-DOFS}

\section{A. Shaking force and shaking moment}

PAMINSA (PArallel Manipulator of the INSA) manipulators belong to a new family of partially decoupled 
parallel robots having between three and six DOFs, which was recently developed at the I.N.S.A. of Rennes (France) [24-27]. The particularity of this family of manipulators is the decoupling of the displacements of the mobile platform in the horizontal plane from the translations along the vertical axis.

Fig. 1a shows a CAD model of a new PAMINSA architecture with 6 DOFs. The parallel structure of this manipulator has only three DOF for displacements of the platform along the $\boldsymbol{x}, \boldsymbol{y}$ and $\boldsymbol{z}$ axes. With regard to the orientation of the end-effector, a serial wrist with 3 DOF is mounted on the platform. As in the previous architectures of PAMINSA manipulators, each leg of the PAMINSA is realized by a pantograph mechanism (Fig. 1b) with two input points $A_{i}$ and $B_{i}$, and one output point $C_{i}(i=1,2,3)$. Each input point $A_{i}$ is connected with the revolute actuator $M_{i}$ by means of a prismatic guide mounted on a rotating link. This type of architecture allows the generation of translations in the horizontal plane by use of the rotating actuators $M_{1}$ and $M_{3}$ and the vertical translations by the linear actuator $M_{v}$. Thus, the displacements $(x, y)$ of point $P$ of the platform in the horizontal plane $\boldsymbol{O} x \boldsymbol{y}$ (respectively the translations along the $\boldsymbol{x}$ - and $\boldsymbol{y}$-axes) are independent of the vertical translations $\boldsymbol{z}$.

This implies that kinematic models controlling the displacement of the manipulator can be divided into two parts:

- one model for the displacements in the horizontal plane (Fig. 2a); this model is equivalent to a $3-\underline{R} P R$ manipulator with two actuated revolute joints and two parallel legs. On this drawing, the displacement of pairs $M_{i}^{\prime}$ (or $H_{i}^{\prime}$ ) corresponds to the displacement of both passive pairs $M_{i}$ (or $H_{i}$ ) and the pantograph linkage;

- one model for the translations along the vertical axis (Fig. $2 b)$ equivalent to the model for the vertical translations of a pantograph linkage ( $k$ is the magnification factor of the pantograph linkage).

The advantages of such a design concept have been discussed in [26, 27].

The PAMINSA manipulators are aimed at high-load carrying applications, but could also be used in different applications, such as pick-and-place or micro-manipulation (as long as the magnification factor of the pantograph linkages does not scale up the displacements but, on the contrary, scales down the movement).

As depicted above, the parallel structure has only three translational DOFs. In order to ensure the three orientational DOFs, a serial wrist is added to the platform (Fig. 3). This wrist has three rotations which can be represented by expressing the direction cosines in terms of $\boldsymbol{z}_{0}-\boldsymbol{x}_{1}-z_{2}$ Euler angles $\phi, \psi, \theta$. This wrist will be used in order to balance the shaking moments during the displacements of the structure.

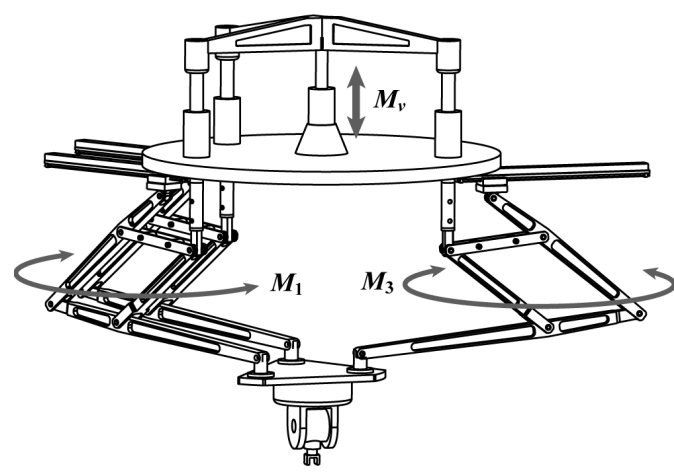

(a)

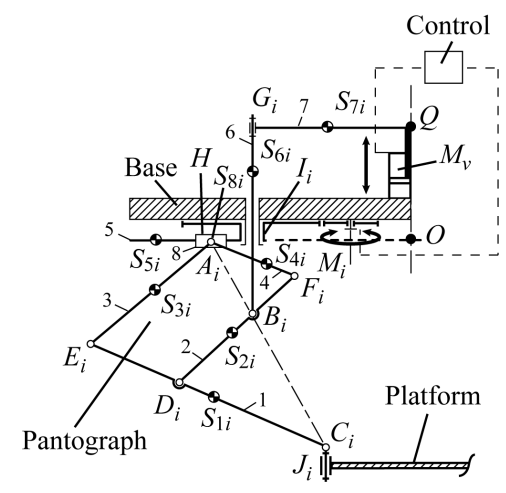

(b)

Fig. 1. The hybrid PAMINSA manipulator. (a) PAMINSA with 6 DOF. (b) Kinematic chain of each leg of the PAMINSA structure

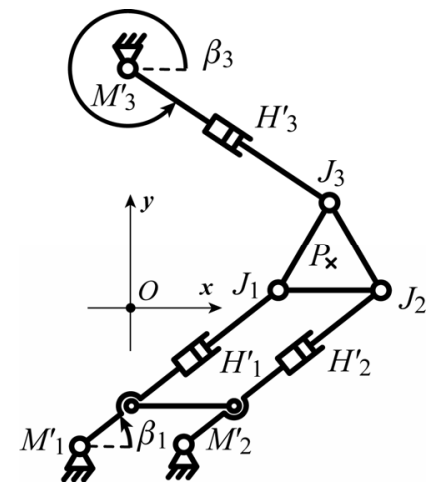

(a)

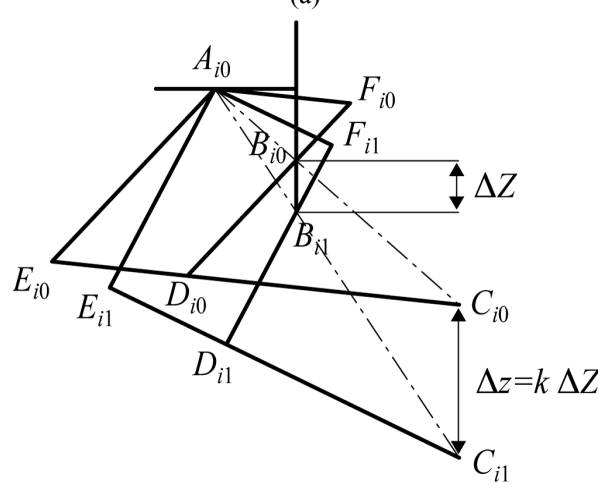

(b)

Fig. 2. Kinematic models for the manipulator under study. (a) Equivalent model for planar movements. (b) Equivalent model for vertical translations 


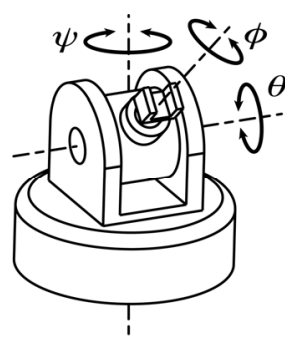

(a)

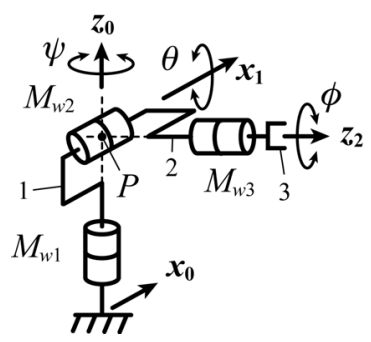

(b)
Fig. 3. The 3-DOF serial wrist mounted on the platform. (a) CAD view. (b) Kinematic model.

The positions of the centers of masses $S_{j i}$ of the links $(j=$ 1 to 8 and $i=1,2,3$ ) are considered at the middle of each link. Moreover, it is assumed that the centers of masses $S_{w i}$ of the wrist links are located at point $P$.

Therefore, for this mechanism, the shaking force $\mathbf{F}$ and shaking moment $\mathbf{M}$ applied on the base can be computed using the general equations:

$$
\begin{aligned}
& \mathbf{F}=m_{p l} \boldsymbol{\Gamma}_{P}+\sum_{i=1}^{3} \sum_{j=1}^{8} m_{j i} \boldsymbol{\Gamma}_{S j i} \\
& \mathbf{M}=m_{p l} \mathbf{O P} \times \boldsymbol{\Gamma}_{P}+\sum_{i=1}^{3} \sum_{j=1}^{8}\left(\mathbf{M}_{j i}^{\mathrm{rot}}+m_{j i} \mathbf{O S} \mathbf{S}_{j i} \times \boldsymbol{\Gamma}_{S j i}\right)+\mathbf{M}_{w r i s t}^{\mathrm{rot}} \\
& \text { with } \mathbf{M}_{j i}^{\mathrm{rot}}=\dot{\mathbf{I}}_{j i} \mathbf{\Omega}_{j i}+\mathbf{I}_{j i} \dot{\mathbf{\Omega}}_{j i} \\
& \text { and } \mathbf{M}_{w r i s t}^{\mathrm{rot}}=\sum_{i=1}^{3} \dot{\mathbf{I}}_{w i} \mathbf{\Omega}_{w i}+\mathbf{I}_{w i} \dot{\mathbf{\Omega}}_{w i}
\end{aligned}
$$

where

- $m_{p l}$ represents the mass of the platform with the wrist;

- $\Gamma_{P}$ represents the acceleration of the end-effector;

- $m_{j i}$ and $\mathbf{I}_{j i}$, represent the mass and the inertia matrix (expressed in the base frame) of the $j$-th limb of leg $i$, respectively;

- $\Gamma_{S j i}$ represents the acceleration of the centre of masses of the $j$-th limb of leg $i$;

- $\Omega_{j i}$ is the vector of rotational velocities of the $j$-th limb of leg $i$;

- $\mathbf{I}_{w i}$ represents the inertia matrix expressed in the base frame of the $i$-th link of the wrist;

- $\boldsymbol{\Omega}_{w i}$ is the vector of rotational velocities of the $i$-th link of the wrist.

Developing and simplifying (1), the expression of the force $\mathbf{F}$ can be rewritten under the form:

$$
\mathbf{F}=\sum_{i=1}^{3}\left(\left[\begin{array}{ccc}
m_{C i 1} & 0 & 0 \\
0 & m_{C i 1} & 0 \\
0 & 0 & m_{C i 2}
\end{array}\right] \boldsymbol{\Gamma}_{C i}+m_{E i} \boldsymbol{\Gamma}_{E i}+m_{S 5 i} \boldsymbol{\Gamma}_{S 5 i}\right)
$$

where $\Gamma_{C i}, \Gamma_{E i}, \Gamma_{S 5 i}$ represent the acceleration of points $C_{i}, S_{5 i}$ and $E_{i}$, and:

$$
m_{C i 2}=\frac{m_{p l}}{3}+\frac{m_{1 i}}{2}+\frac{2 m_{2 i}+m_{4 i}+2 m_{6 i}+2 m_{7 i}}{2 k}
$$

$$
\begin{aligned}
m_{C i 1}= & \frac{m_{p l}}{3}+\frac{m_{3 i}+m_{4 i}}{2(1-k)}+\frac{m_{1 i}+m_{2 i} / k}{2} \\
& +\frac{m_{2 i}+m_{4 i}+2 m_{8 i}}{2 k(1-k)} \\
m_{E i}= & \frac{m_{1 i}+m_{3 i}}{2}+\frac{(k-1) m_{2 i}}{2 k}-\frac{m_{2 i}+m_{4 i}+2 m_{8 i}}{2 k} \\
m_{S 5 i}= & m_{5 i}
\end{aligned}
$$

where $k=B_{i} F_{i} / D_{i} F_{i}$ is the magnification factor of the pantograph linkage.

In order to estimate the variable dynamic loads transmitted to the base of PAMINSA, we carried out the simulations using ADAMS software. For this purpose, a standard cycle of pick-and-place motion $(25 \times 300 \mathrm{~mm}$ in 0.5 s) for a fifth-order polynomial trajectory with the following initial and final locations $x_{i}=0.48 \mathrm{~m}, x_{f}=0.48 \mathrm{~m}$ and $y_{i}=$ $-0.1 \mathrm{~m}, y_{f}=0.2 \mathrm{~m}$ with constant orientation of the wrist equal to zero. The geometric and inertia parameters of the robot are the following:

- parameters for the position of points $M_{i}$ and $J_{i}: a=0.1 \mathrm{~m}$, $b=0.175 \mathrm{~m}, c=0.42 \mathrm{~m}, d=0.1 \mathrm{~m}, e=0.05 \mathrm{~m}$ and $f=$ $0.12 \mathrm{~m}$;

- magnification factor of the pantograph: $k=3$;

- lengths of the links of the pantograph linkages: $B_{i} G_{i}=$ $0.442 \mathrm{~m}, A_{i} E_{i}=D_{i} F_{i}=0.42 \mathrm{~m}, C_{i} E_{i}=k A_{i} F_{i}=0.63 \mathrm{~m}, C_{i} J_{i}$ $=0.0275 \mathrm{~m}, M_{i} S_{5 i}=0.15 \mathrm{~m}, M_{i} O_{i}=0.308 \mathrm{~m}$;

- mass of the platform with the wrist: $m_{p l}=4.41 \mathrm{~kg}$;

- masses of the links of the pantograph linkages: $m_{1 i}=0.622 \mathrm{~kg}, m_{2 i}=m_{3 i}=0.406 \mathrm{~kg}, m_{4 i}=0.107 \mathrm{~kg}, m_{5 i}=$ $0.436 \mathrm{~kg}, m_{6 i}=0.921 \mathrm{~kg}, m_{7 i}=1.2 \mathrm{~kg}, m_{8 i}=0.305 \mathrm{~kg}$;

- terms of the inertia matrices of the links of the pantograph linkages:

$J_{X X}^{(1)}=0.0012 \mathrm{~kg} / \mathrm{m}^{2}, J_{Y Y}^{(1)}=0.048 \mathrm{~kg} / \mathrm{m}^{2}, J_{Y Y}^{(5)}=0.02 \mathrm{~kg} / \mathrm{m}^{2}$,

$J_{X X}^{(5)}=0.0006 \mathrm{~kg} / \mathrm{m}^{2}, J_{X X}^{(2)}=J_{X X}^{(3)}=0.0038 \mathrm{~kg} / \mathrm{m}^{2}$,

$J_{Y Y}^{(2)}=J_{Y Y}^{(3)}=0.02 \mathrm{~kg} / \mathrm{m}^{2}, J_{X X}^{(4)}=0.0008 \mathrm{~kg} / \mathrm{m}^{2}$,

$J_{Y Y}^{(4)}=0.003 \mathrm{~kg} / \mathrm{m}^{2}, J_{X X}^{(6)}=0.003 \mathrm{~kg} / \mathrm{m}^{2}$,

$J_{Y Y}^{(6)}=0.071 \mathrm{~kg} / \mathrm{m}^{2}$.

- terms of the inertia matrices of the links of the wrist:

$J_{X X}^{(w 1)}=0.019 \mathrm{~kg} / \mathrm{m}^{2}, J_{Y Y}^{(w 1)}=0.337 \mathrm{~kg} / \mathrm{m}^{2}$,

$J_{X X}^{(w 2)}=0.039 \mathrm{~kg} / \mathrm{m}^{2}, J_{Y Y}^{(w 2)}=2.63 \mathrm{~kg} / \mathrm{m}^{2}$,

$J_{X X}^{(w 3)}=0.088 \mathrm{~kg} / \mathrm{m}^{2}, J_{Y Y}^{(w 3)}=3.35 \mathrm{~kg} / \mathrm{m}^{2}$.

Fig. 4 shows the shaking force and moment variations of the unbalanced robot computed with ADAMS (in solid line).

\section{B. Shaking force and shaking moment balancing}

The shaking force can be balanced by cancelling the terms $m_{C i 1}, m_{C i 2}, m_{S 5 i}$ and $m_{E i}$. This can be achieved by the redistribution of the centres of masses of the moving links, i.e.:

- for the cancellation of terms $m_{C i 1}$ and $m_{E i}$, by the addition of counterweights $m_{C P 1 i}$ and $m_{C P 2 i}$ positioned, by example, at points $A_{i}$ and $F_{i}$ respectively, 


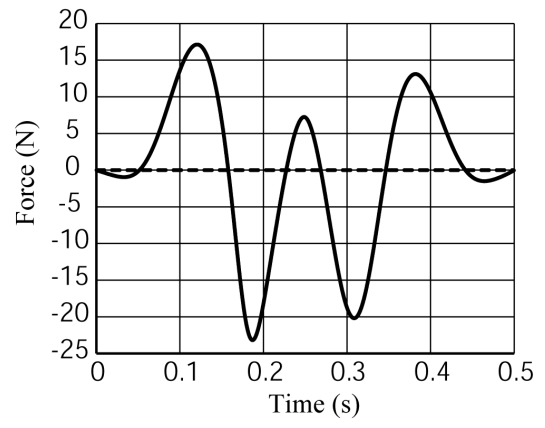

(a)

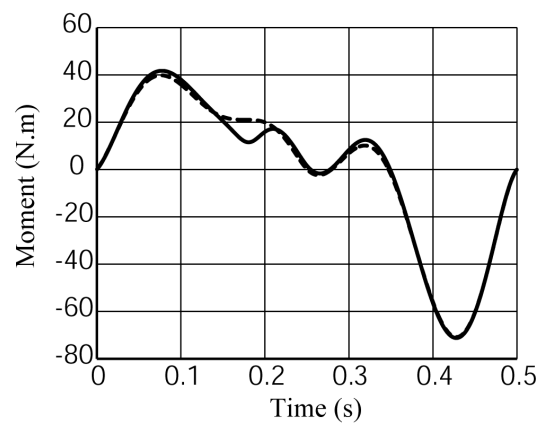

(d)

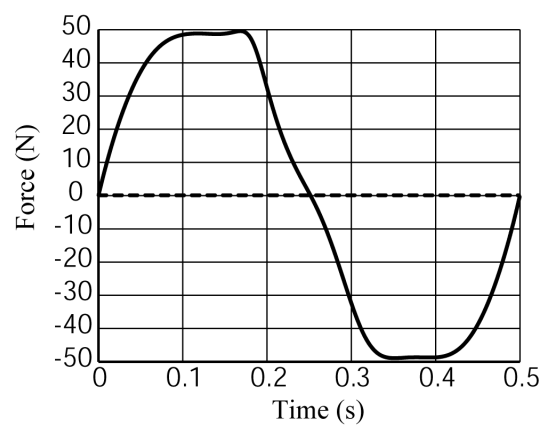

(b)

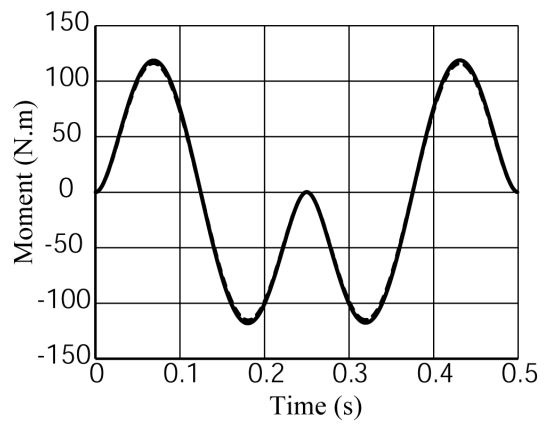

(e)

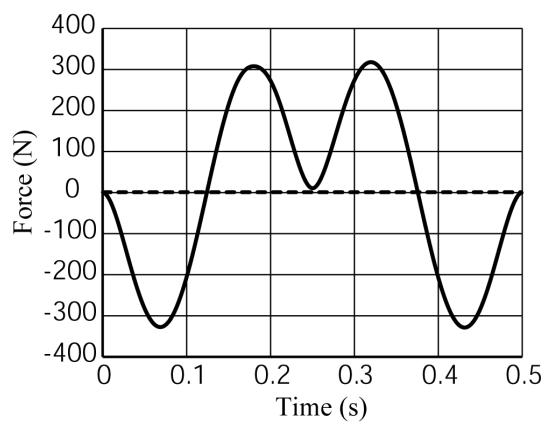

(c)

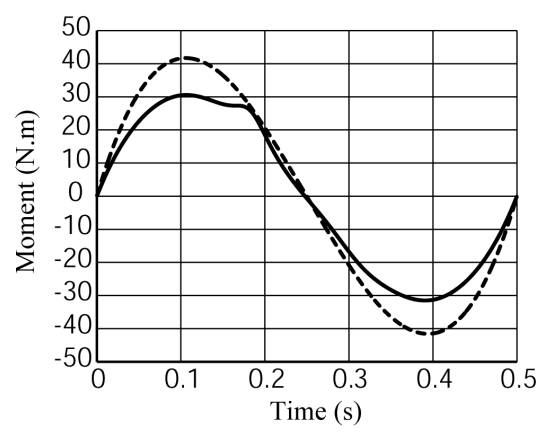

(f)

Fig. 4 Shaking force before (solid line) and after (dashed line) balancing and shaking moment before (solid line) and after force-balancing (dashed line). (a) Shaking force along $\boldsymbol{x}$-axis. (b) Shaking force along $\boldsymbol{y}$-axis. (c) Shaking force along $\boldsymbol{z}$-axis. (c) Shaking moment around $\boldsymbol{x}$-axis. (e) Shaking moment around $y$-axis. (f) Shaking moment around $z$-axis.

- for canceling the effects of term $m_{S 5 i}$, by adding a counterweight $m_{C P 3 i}$ on the link $5_{i}$ positioned, by example, at points $L_{i}$ such as $\mathbf{O L}_{i}=\mathbf{O} \mathbf{O}_{i}+r \mathbf{O}_{i} \mathbf{S}_{5 i}, r<0$.

- for canceling the effects of term $m_{C i 2}$, by adding counterweights undergoing similar but opposite movements to the end-effector motion.

With the added counterweights and the supplementary system, equations (6) to (9) can be rewritten as:

$$
\begin{aligned}
m_{C i 1}= & \frac{m_{p l}}{3}+\frac{m_{3 i}+m_{4 i}+2 m_{C P 1 i}}{2(1-k)}+\frac{m_{1 i}+m_{2 i} / k}{2} \\
& +\frac{m_{4 i}+m_{2 i}+2 m_{8 i}+2 m_{C P 2 i}}{2 k(1-k)} \\
m_{C i 2}= & \frac{2 m_{2 i}+m_{4 i}+2 m_{6 i}+2 m_{7 i}+2 m_{C P 2 i}}{2 k}+\frac{m_{p l}}{3}+\frac{m_{1 i}}{2} \\
m_{E i}= & \frac{m_{1 i}+m_{3 i}}{2}+\frac{(k-1) m_{2 i}}{2 k}-\frac{m_{2 i}+m_{4 i}+2 m_{8 i}+2 m_{C P 2 i}}{2 k} \\
m_{S 5 i}= & m_{5 i}+r m_{C P 5 i}
\end{aligned}
$$

which leads to the expressions of the masses of the counterweights:

$$
\begin{aligned}
m_{C P 1 i}= & (k-1)\left(\frac{m_{3 i}+m_{4 i}}{2(1-k)}+\frac{m_{1 i}+m_{2 i} / k}{2}+\frac{m_{p l}}{3}\right) \\
& -\frac{m_{4 i}+m_{2 i}+2 m_{8 i}+2 m_{C P 2 i}}{2 k} \\
m_{C P 2 i} & =k\left(\frac{m_{1 i}+m_{3 i}}{2}+\frac{(k-1) m_{2 i}}{2 k}-\frac{m_{2 i}+m_{4 i}+2 m_{8 i}}{2 k}\right)
\end{aligned}
$$

$m_{C P 3 i}=-m_{5 i} / r$.

The effects of counterweights on shaking force and shaking moment are shown in Fig. 4 (with a dashed line). For the manipulator under study, the masses of the counterweights are $m_{C P 1 i}=4.49 \mathrm{~kg}, m_{C P 2 i}=1.69 \mathrm{~kg}$, and $m_{C P 3 i}=0.436 \mathrm{~kg}$ (taking into account that $r=-1$ ). Note that, although the added masses allow cancelling of the shaking force, there is an increase of about $35 \%$ in the shaking moment around the $z$-axis. Concerning the shaking moment around $\boldsymbol{x}$ and $\boldsymbol{y}$ axes, the values before and after mass balancing are similar.

After force balancing, the expression of the shaking moment $\mathbf{M}^{\text {bal }}$ becomes:

$$
\begin{aligned}
\mathbf{M}^{\text {bal }}= & \mathbf{M}+\sum_{i=1}^{3}\left(m_{C P 1 i} \mathbf{O A}_{i} \times \Gamma_{A i}+m_{C P 2 i} \mathbf{O F _ { i }} \times \Gamma_{F i}\right) \\
& +\sum_{i=1}^{3}\left(m_{C P 3 i} \mathbf{O L}_{i} \times \Gamma_{L i}\right)
\end{aligned}
$$

The expression of $\mathbf{M}$ is given in equation (2).

The term $\mathbf{M}$ of equation (17) depends on a free parameter $\mathbf{M}_{\text {wrist }}^{\text {rot }}$, which defines the orientations of the end-effector between its initial and final positions, but which does not have any influence on the prescribed trajectory.

Thus, we have to adjust the rotations of the wrist in order to cancel the shaking moment. However, according to equation (17), which is nonlinear, the expressions of the rotations are difficult to compute. Therefore it is preferable to compute the values of the rotation angles starting from the 
expression of the angular momentum $\mathbf{H}$ of the mechanism. Thus, $\mathbf{H}$ can be written in the following form:

$$
\mathbf{H}=\mathbf{D}+\mathbf{H}_{\text {wrist }}^{\text {rot }}
$$

with $\mathbf{D}$ and $\mathbf{H}_{\text {wrist }}^{\text {rot }}$ the angular momentums of PAMINSA and of the wrist, respectively.

$$
\begin{aligned}
\mathbf{D} & =m_{p l} \mathbf{O P} \times \mathbf{V}_{P}+\sum_{i=1}^{3} \sum_{j=1}^{8}\left(\mathbf{I}_{j i} \boldsymbol{\Omega}_{j i}+m_{j i} \mathbf{O} \mathbf{S}_{j i} \times \mathbf{V}_{S j i}\right) \\
& +\sum_{i=1}^{3}\left(m_{C P 1 i} \mathbf{O} \mathbf{A}_{j i} \times \mathbf{V}_{A j i}+m_{C P 2 i} \mathbf{O} \mathbf{F}_{j i} \times \mathbf{V}_{F j i}\right) \\
& +\sum_{i=1}^{3}\left(m_{C P 3 i} \mathbf{O} \mathbf{L}_{i} \times \mathbf{V}_{L i}\right) \\
\mathbf{H}_{w r i s t}^{\mathrm{rot}} & =\sum_{i=1}^{3} \mathbf{I}_{w i} \boldsymbol{\Omega}_{w i}
\end{aligned}
$$

where

- $\mathbf{V}_{P}$ represents the velocity of the end-effector,

- $\mathbf{V}_{S j i}$ represents the velocity of the centre of masses of the $j$-th limb of leg $i$.

For reason of brevity, their expressions are not given in here.

In order to cancel the shaking moment of the mechanism, its angular moment $\mathbf{H}$ has to be constant. Thus, considering that $\mathbf{H}$ should be equal to zero, the following condition will be achieved:

$$
\mathbf{H}_{\text {wrist }}^{\text {rot }}=-\mathbf{D}
$$

Simplifying the expression of $\mathbf{H}_{\text {wrist }}^{\mathrm{rot}}$, one can rewrite this term as a linear combination of the angular velocities of the actuators of the wrist:

$$
\mathbf{H}_{w r i s t}^{\mathrm{rot}}=\mathbf{N}\left[\begin{array}{lll}
\dot{\theta} & \dot{\phi} & \dot{\psi}
\end{array}\right]^{T}
$$

with

$$
\mathbf{N}=\left[\begin{array}{cc}
\mathbf{0}_{2 \times 2} & \mathbf{0}_{2 \times 1} \\
\mathbf{0}_{1 \times 2} & 1
\end{array}\right] \mathbf{I}_{w 1}+\left(\mathbf{I}_{w 2}+\mathbf{I}_{w 3} \mathbf{E}_{3}\right)\left[\begin{array}{ccc}
1 & 0 & 0 \\
0 & 0 & 0 \\
0 & 0 & 1
\end{array}\right]+\mathbf{I}_{w 3}\left[\begin{array}{cc}
\mathbf{0}_{1 \times 2} & 0 \\
\mathbf{0}_{1 \times 2} & 1 \\
\mathbf{0}_{1 \times 2} & 0
\end{array}\right]^{T}
$$

where $\mathbf{I}_{w i}$ is the inertia matrix of the $i$-th link of the wrist, $\mathbf{0}_{i \times j}$ corresponds to the zero matrix with $i$ lines and $j$ columns, $\mathbf{E}_{\mathbf{3}}$ is a rotation matrix:

$$
\mathbf{E}_{3}=\boldsymbol{\operatorname { R o t }}(\phi, z) \operatorname{Rot}(\theta, \boldsymbol{x}) \text {. }
$$

Thus, by introducing equation (23) into relation (21), it is possible to determine the trajectory of the wrist, which can cancel the angular momentum, and as a result, the shaking moment:

$$
[\dot{\theta}, \dot{\phi}, \dot{\psi}]^{T}=-\mathbf{N}^{-1} \mathbf{D}
$$

Taking into account that matrix $\mathbf{N}$ depends on the angles of rotation of the wrist, the value of $\theta, \phi$ and $\psi$ at time $t$ can be found by a numerical integration:

$$
\left[\begin{array}{l}
\theta(t) \\
\phi(t) \\
\psi(t)
\end{array}\right]=\left[\begin{array}{l}
\theta(t-\delta t) \\
\phi(t-\delta t) \\
\psi(t-\delta t)
\end{array}\right]+\left[\begin{array}{c}
\dot{\theta}(t-\delta t) \\
\dot{\phi}(t-\delta t) \\
\dot{\psi}(t-\delta t)
\end{array}\right] \delta t
$$

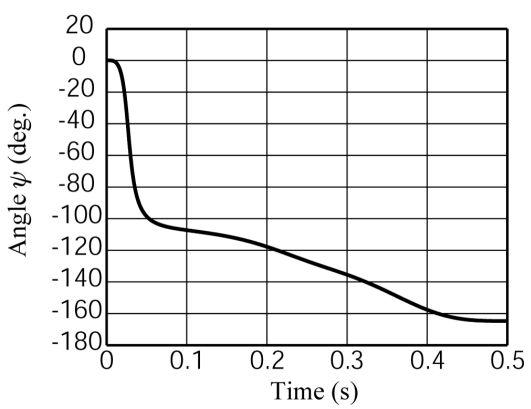

(a)

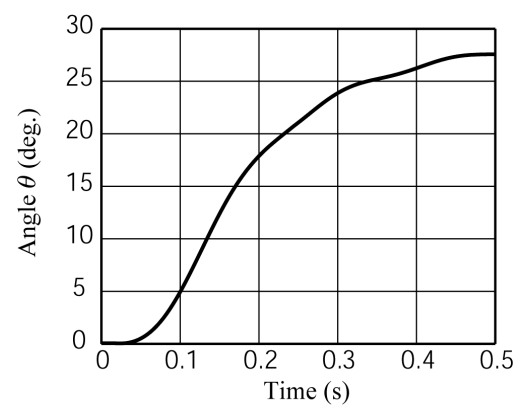

(b)

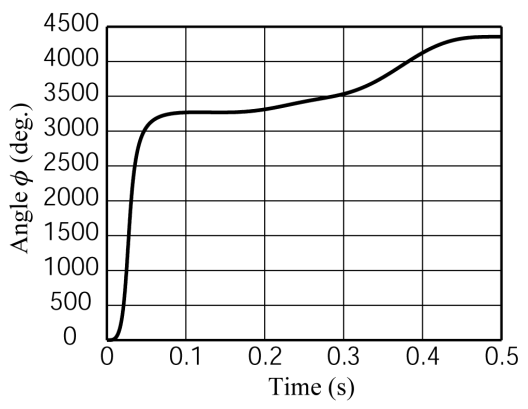

(c)

Fig. 5. Rotations of the wrist used for canceling the shaking moment. (a) Angle $\psi$. (b) Angle $\theta$. (c) Angle $\phi$.

where $\delta t$ corresponds to the integration step, and the values of the angles and angular velocities at time $t-\delta t$ are considered known.

For the examined manipulator, we determine the required rotations of the end-effector, which makes it possible to cancel shaking moment (Fig. 5).

It should be noted that the moment of inertia of link 3 of the wrist is relatively small compared to the moments of inertia of the robot links. Thus, to ensure the balancing of the shaking moment, it is necessary that it makes several full revolutions during the motion of the robot in the horizontal plane.

Finally, note that in practice there are small deviations from the ideal zero reaction. Sources of such deviations include manufacturing errors, friction and clearances in the joints and drive actuators, as well as manipulator unbalancing due to a payload. However, in all of these cases, the proposed balancing method will eliminate the most significant base reactions due to the accelerating links of the robot. 


\section{CONCLUSION}

A new field of shaking force and shaking moment balancing is in the development of fast parallel manipulators, which are very efficient for advanced robotic applications. In this paper, a shaking force and shaking moment balancing solution is developed for the positionorientation decoupled PAMINSA manipulator. The shaking forces of the manipulator in the horizontal plane are eliminated via optimum redistribution of masses, making the total mass center of the moving links stationary. With regard to the vertical inertia forces, the opposite motion of the endeffector and counterweights is applied. The shaking moment on the frame of the manipulator are eliminated by optimal control of the decoupled orientation motion. Numerical simulations carried out using ADAMS software show that after such a balancing, the manipulator transmits no inertia loads to its surroundings, i.e., the sum of all ground bearing forces and their moments are eliminated.

\section{REFERENCES}

[1] G.G. Lowen, F.R. Tepper, and R.S. Berkof, "Balancing of linkages an update," Mechanism and Machine Theory, Vol. 18, No. 3, 1983, pp. $213-230$.

[2] V. Arakelian, and M. Smith, "Shaking force and shaking moment balancing of mechanisms: an historical review with new examples," Journal of Mechanical Design, Vol. 127, March, pp. 334-339 (see also ERRATUM, Vol. 127, September, 2005, pp. 1034-1035).

[3] Arakelian V., Dahan M., Smith M.R., 2000, "A historical review of the evolution of the theory on balancing of mechanisms", International Symposium on History of Machines and Mechanisms Proceedings HMM2000, Kluwer Academic Publishers, Dordrecht/ Boston/London, 2005, pp. 291-300.

[4] S.K. Agrawal, and A. Fattah, "Reactionless space and ground robots: novel designs and concept studies," Mechanism and Machine Theory, Vol. 39, No. 1, 2004, pp. 25-40.

[5] J. Wang, and C.M. Gosselin, "Static balancing of spatial three-degreeof-freedom parallel mechanisms," Mechanism and Machine Theory, Vol. 34, No. 3, 1999, pp. 437-452.

[6] Newman, W.S. and Hogan, N., 1986, "The optimal control of balanced manipulators," Proc. winter annual meeting of the ASME, Anaheim, California.

[7] T. Laliberté, C.M. Gosselin, and M. Jean, "Static balancing of 3-DOF planar parallel mechanisms," IEEE/ASME Transactions on Mechatronics, Vol. 4, No. 4, 1999, pp. 363-377.

[8] K. Fujikoshi, "Balancing apparatus for jointed robot," Japanese Patent JP51-122254, October 26, 1976.

[9] J. Wang, and C.M. Gosselin, "Static balancing of spatial four-degreeof-freedom parallel mechanisms," Mechanism and Machine Theory, Vol. 35, No. 4, 2000, pp. 563-592.

[10] A. Russo, R. Sinatra, and F. Xi, "Static balancing of parallel robots," Mechanism and Machine Theory, Vol. 40, No. 2, 2005, pp. 191-202.

[11] R.S. Berkof, "Complete fore and moment balancing of inline four-bar linkages," Mechanism and Machine Theory, Vol. 8, No. 3, 1973, pp. $397-410$.

[12] V., Arakelian, and M. Smith, "Complete shaking force and shaking moment balancing of linkages," Mechanism and Machine Theory, Vol. 34, No. 8, 1999, pp. 1141-1153.

[13] H. Dresig, S. Naake, and L. Rockausen, "Vollständiger und harmonischer Ausgleich ebener Mechanismen," VDI Verlag, Düsseldorf, 1994.

[14] J.L. Herder, and C.M. Gosselin, "A counter-rotary counterweight for light-weight dynamic balancing," Proc. ASME 2004 DETC/CIEC Conference, Salt Lake City, Utah, USA, September 28 - October 2, 2004, pp. 659-667.
[15] I.S. Kochev, "General theory of complete shaking moment balancing of planar linkages: a critical review," Mechanism and Machine Theory, Vol. 35, No. 11, 2000, pp. 1501-1514.

[16] R. Ricard, and C.M. Gosselin, "On the development of reactionless parallel manipulators," Proc. ASME 2000 DETC, Baltimore, Maryland, USA, September 10-13, 2000.

[17] Y. Wu, and C.M. Gosselin, "On the synthesis of a reactionless 6-DOF parallel mechanism using planar four-bar linkages," Proc. Workshop on Fundamental Issues and Future Research Directions for Parallel Mechanisms and Manipulators, Quebec City, Quebec, Canada, October 3-4, 2002.

[18] C.M. Gosselin, G. Côté, and Y. Wu, "Synthesis and design of reactionless three-degree-of-freedom parallel mechanisms," IEEE Transaction on Robotics and Automation, Vol. 20, No. 2, April, 2004, pp. 191-199.

[19] S. Foucault, and C.M. Gosselin, "Synthesis, design, and prototyping of a planar three degrees-of-freedom reactionless parallel mechanism," Journal of Mechanical Design, Vol. 126, November, 2004, pp. 992999.

[20] Y. Wu, and C.M. Gosselin, "Design of reactionless 3-DOF and 6-DOF parallel manipulators using parallelepiped mechanisms," IEEE Transaction on Robotics and Automation, Vol. 21, No. 5, October, 2005, pp. 821-833.

[21] E. Papadopoulos, and A. Abu-Abed, "Design and motion planning for a zero-reaction manipulator," Proc. IEEE International Conference on Robotics and Automation (ICRA), San Diego, CA, USA, 1994, pp. 1554-1559.

[22] A. Fattah, and S.K. Agrawal, "On the design of reactionless 3-DOF planar parallel mechanisms," Mechanism and Machine Theory, Vol. 41, No. 1, 2006, pp. 70-82.

[23] V. Arakelian and S. Briot, "Dynamic Balancing of the Scara Robot," The 17-th CISM-IFToMM Symposium on Robot Design, Dynamics and Control (RoManSy 2008), July 5-9, Tokyo, Japan, 2008, pp. 167174.

[24] S. Briot, "Analysis and optimization of a new family of parallel manipulators with decoupled motions," Ph.D. Thesis, INSA, Rennes, France, 2007.

[25] V. Arakelian, S. Briot, S. Guegan, and J. Le Flecher, "Design and prototyping of new 4-, 5- and 6-DOF parallel manipulators based on the copying properties of the pantograph linkage," Proc. 36th International Symposium on Robotics (ISR), Tokyo, Japan, November 29 - December 1, 2005.

[26] V. Arakelian, P. Maurine, S. Briot, and E. Pion, "Parallel robot comprising means for setting in motion a mobile element split in two separate subassemblies," International Patent WO 2006/021629, January 27, 2006.

[27] S. Briot, V. Arakelian, and S. Guegan, " PAMINSA: A new family of partially decoupled parallel manipulators," Mechanism and Machine Theory, Vol. 44, No. 2, pp. 425-444. 